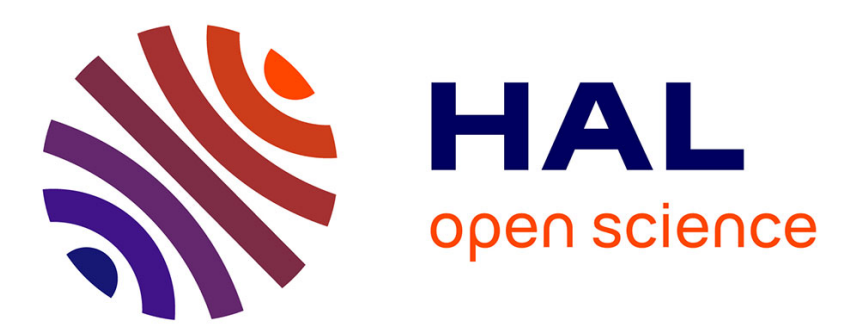

\title{
Finite element approximation of elliptic partial differential equations on implicit surfaces
}

Martin Burger

\section{To cite this version:}

Martin Burger. Finite element approximation of elliptic partial differential equations on implicit surfaces. Computing and Visualization in Science, 2009, 12 (3), pp.87-100. 10.1007/s00791-007-0081x . hal-01742391

\section{HAL Id: hal-01742391 \\ https://hal.science/hal-01742391}

Submitted on 24 Mar 2018

HAL is a multi-disciplinary open access archive for the deposit and dissemination of scientific research documents, whether they are published or not. The documents may come from teaching and research institutions in France or abroad, or from public or private research centers.
L'archive ouverte pluridisciplinaire HAL, est destinée au dépôt et à la diffusion de documents scientifiques de niveau recherche, publiés ou non, émanant des établissements d'enseignement et de recherche français ou étrangers, des laboratoires publics ou privés. 


\title{
Martin Burger \\ Finite Element Approximation of Elliptic Partial Differential Equations on Implicit Surfaces
}

\begin{abstract}
The aim of this paper is to investigate finite element methods for the solution of elliptic partial differential equations on implicitely defined surfaces. The problem of solving such equations without triangulating surfaces is of increasing importance in various applications, and their discretization has recently been investigated in the framework of finite difference methods. For the two most frequently used implicit representations of surfaces, namely level set methods and phase-field methods, we discuss the construction of finite element schemes, the solution of the arising discretized problems, and provide error estimates. The convergence properties of the finite element methods are illustrated by computations for several test problems.
\end{abstract}

\section{Introduction}

The solution of partial differential equations on curves and surfaces has received growing interest in the last years due to a variety of applications in computer graphics (cf. $[15,16,26]$ ), materials science (cf. $[9,13,18]$ ), and inverse problems (cf. $[8,19,20])$. In many of these applications, elliptic or parabolic partial differential equations have to be solved on varying geometries, which means that after time-discretization a large number of elliptic problems remain to be solved numerically on different curves or surfaces. The standard approach to discretize these elliptic problems are finite element methods on curve or surface triangulations, which can cause a tremendous computational effort for varying geometries, since the triangulation has to be recomputed for each change in the geometry. Moreover, the approximation properties of such triangulations are not yet well understood except for simple geometries, and it is not clear how higher order geometric characteristics such as curvatures are to be represented on the triangulation.

In order to avoid surface triangulations, it seems reasonable to consider Eulerian methods based on implicit surface representations, which were used with great success in the construction of computational schemes for evolving curves and surfaces. By now the two standard Eulerian approaches for evolving surfaces are level set methods (cf. e.g. [25,24]) and phase-field methods (cf. e.g. [3,11]). Level set methods are based on an extension of the equation on the surface to all level sets of an implicit representation function, ideally the signed distance function. Phase-field methods approximate the equation by a parameter-dependent reaction-diffusion equation, whose solution is an approximation of a rescaled signed distance function.

Martin Burger

Industrial Mathematics Institute, Johannes Kepler Universität. Altenbergerstr. 69, A 4040 Linz, Austria Tel.: $++43-732-24689224$

Fax: ++43-732-2468 8855

E-mail: martin.burger@jku.at. 
Eulerian schemes based on a level set representation of the surface have been proposed recently by Bertalmio et. al. [4]. The starting point of their analysis is an implicit representation of the surface, i.e., as the zero level set of a continuous function $\phi: \mathbb{R}^{d} \rightarrow \mathbb{R}$ :

$$
\Sigma=\left\{x \in \mathbb{R}^{d} \mid \phi(x)=0\right\} .
$$

The basic idea of the approach in [4] is to rewrite the derivatives with respect to surface variables as projections of derivatives in $\mathbb{R}^{d}$ (with projection operators defined in terms of the level set function), and to extend the equations to a set $D$ of positive Lebesgue measure in $\mathbb{R}^{n}$. In particular, the surface gradient can be written as

$$
\nabla_{S} u=P \nabla u, \quad \text { with } P=I-\frac{\nabla \phi \otimes \nabla \phi}{|\nabla \phi|^{2}}
$$

Using extensions of the coefficients, one can then extend the partial differential equation to all level sets

$$
\Sigma^{\alpha}=\left\{x \in \mathbb{R}^{d} \mid \phi(x)=\alpha\right\}
$$

for all $|\alpha|$ sufficiently small, and thus, to a neighbourhood of $\Sigma$. The implicit dependence on $\Sigma$ is then replaced by a dependence on $\nabla \phi$. The benefit of this extension is the fact that standard discretization methods can be used in a small domain $D$, and therefore no surface triangulations are needed. The numerical methods in [4] and subsequent work (cf. [5,21,22]) were developed for the solution of time-dependent parabolic and hyperbolic equations, using explicit time-stepping. Stationary elliptic equations such as e.g. the Laplace-Beltrami equation, which appear in many applications either of interest for themselves (cf. e.g. $[15,16,19,26]$ ) or as a subproblem in some larger system of equations (cf. e.g. $[2,9,10,12,18,20]$ ) have not yet been investigated. Moreover, all the schemes developed so far were based on finite differences on parts of a regular grid, while finite element methods allowing much more flexibility with respect to the grid have not yet been investigated in this respect.

Phase-field methods for equations on surfaces have not yet been investigated in detail, such approaches are so far only considered as subproblems in the second-order splitting of fourthorder equations, with few rigorous arguments as the phase-field parameter tends to zero. The fundamentals of a phase-field representation are a phase-field function $\psi$, asymptotically of the form

$$
\psi(x)=p\left(\frac{d(x)}{\epsilon}\right)+\epsilon q(x)+\mathcal{O}\left(\epsilon^{2}\right),
$$

where $d$ denotes the signed distance function to $\Sigma, \epsilon>0$ is a small parameter, and $p$ is a monotone function such that $p(-\infty)=-1$ and $p(+\infty)=1$. Prototypes for the function $p$ are

$$
p(s)=\frac{1}{\pi} \arctan (\gamma s) \quad \text { or } \quad p(s)=\frac{2}{1+\exp (-s)}-1 .
$$

The first order expansion satisfies $q=0$ on $\Sigma,\|q\|_{\infty}=\mathcal{O}(1)$, and $\|\nabla q\|_{\infty}=\mathcal{O}(1)$.

In this paper, we shall investigate Eulerian methods for linear elliptic equations of the form

$$
-\operatorname{div}_{S}\left(a \nabla_{S} u\right)+c u=f, \quad \text { on } \Sigma
$$

where $\operatorname{div}_{S}$ and $\nabla_{S}$ denote the surface divergence and gradient, respectively. The two extensions and approximations of the equation on a domain $D \supset \Sigma$ we use are

- Level set representation: For an implicit representation with level set function $\phi$, we can follow the formal argumentation given in [4] and extend (1.4) as

$$
-\frac{1}{|\nabla \phi|} \operatorname{div}(|\nabla \phi| \hat{a}(., \phi) P \nabla \hat{u})+\hat{c}(., \phi) \hat{u}=\hat{f}(., \phi),
$$


with $\hat{a}, \hat{c}$, and $\hat{f}$ being appropriate extensions of the coefficients $a, c$, and $f$ in (1.4). In order to obtain a consistent extension, one needs that

$$
\hat{a}(x, 0)=a(x), \quad \hat{c}(x, 0)=c(x), \quad \hat{f}(x, 0)=f(x), \quad \forall x \in \Sigma .
$$

- Phase-field representation: For an implicit representation with a phase-field function $\psi$, an approximation of (1.4) is given by

$$
-\operatorname{div}\left(\left(1-\psi^{2}\right) \tilde{a}(., \psi) \nabla v\right)+\left(1-\psi^{2}\right) \tilde{c}(., \psi) v=\left(1-\psi^{2}\right) \tilde{f}(., \psi),
$$

with $\tilde{a}, \tilde{c}$, and $\tilde{f}$ being appropriate extensions of the coefficients $a, c$, and $f$ in (1.4). Consistency of the extension corresponds to the condition

$$
\tilde{a}(x, 0)=a(x), \quad \tilde{c}(x, 0)=c(x), \quad \tilde{f}(x, 0)=f(x), \quad \forall x \in \Sigma .
$$

The phase-field function is assumed to satisfy an expansion of the form (1.3). For simplicity we shall assume below that $\tilde{a}, \tilde{c}$, and $\tilde{f}$ are constant extensions of $a, c$, and $f$ in normal direction, but analogous results can be derived (with more complicated computations) for other sufficiently regular extensions.

Our aim is to provide a definition and analysis of weak solutions for the extension of the equation (1.4), and discuss their approximation by standard finite element methods. In practical applications, one usually encounters two different cases, namely

- $L^{2}$-coercive problems, which denotes the case where $c$ is strictly positive on $\Sigma$ and thus, no additional condition is needed to obtain a unique solution.

- Constrained problems, which denotes the case where $c$ vanishes on a set of positive $d-1$ dimensional Hausdorff-measure on $\Sigma$, and thus, an additional constraint is needed to obtain a unique solution. A typical constraint (e.g. in surface diffusion problems, cf. $[10,12]$ ) acts on the average value, e.g.,

$$
\int_{\Sigma} u d s=0
$$

In this paper we shall mainly consider the first case. The extension to the case of constrained problems is rather straight-forward on the continuous level as we shall see below, but the construction of appropriate finite element spaces for the extended problems turns out to be a very hard problem, which is left to future research.

For simplicity we shall assume throughout the paper that all the coefficients $a, c$, and $f$ are given continuous functions on $D$, but less regular data and nonlinear dependencies could be treated in a similar way. Throughout this paper we shall also assume that $a$ is strictly positive and $c$ is nonnegative.

\section{Methods based on Level Set Representations}

In the following we discuss the variational formulation and finite element approximation of the extended equation (1.5). We shall supplement the equation by an artificial boundary condition

$$
P \nabla u \cdot n=0 \quad \text { on } \partial D .
$$

We start our analysis by introducing Sobolev spaces suitable for the analysis of the extended elliptic operator. 


\subsection{Extended Sobolev Spaces}

The starting point of a variational formulation is the specification of the function spaces we shall use. By $L^{2}(D)$ we shall denote the usual Lebesgue space on $D$, and moreover we introduce the "partial" Sobolev space

$$
H_{P}^{1}:=\left\{u \in L^{2}(\Omega) \mid(P \nabla u) \in L^{2}(\Omega)\right\}
$$

with the scalar product

$$
\langle u, v\rangle_{H_{P}^{1}}=\int_{D}((P \nabla u) \cdot(P \nabla v)+u v) d x .
$$

Note that for any shape with well-defined boundary $\Sigma$, we can choose a function $\phi$ such that $\nabla \phi \in L^{\infty}(\Omega)$ (e.g. the signed distance function, cf. [14]), so that the condition $(P \nabla u) \in L^{2}(\Omega)$ is mainly a regularity condition for $u$. However, in order to define distributional derivatives, a regularity condition on $\Sigma$ is needed. For a test function $\eta \in C_{0}^{\infty}(D)$, we can define

$$
\langle P \nabla u, \eta\rangle:=-\langle u, \operatorname{div}(P \eta)\rangle_{L^{2}}=-\int_{D} u \operatorname{div}(P \eta) d x
$$

In order to obtain a well-defined linear functional of $u$ one has to impose the condition $\operatorname{div}(P \eta) \in L^{2}(D)$, and since $\eta$ is smooth and $\phi \in W^{1, \infty}(D)$, this is equivalent to

$$
\operatorname{div}\left(\frac{\nabla \phi}{|\nabla \phi|} \otimes \frac{\nabla \phi}{|\nabla \phi|}\right) \in L^{2}(D)^{d}
$$

which we shall assume below as a regularity condition on the surface and on the level set function. Note that this is a very weak regularity assumption, in the case of curves we have

$$
\operatorname{div}\left(\frac{\nabla \phi}{|\nabla \phi|} \otimes \frac{\nabla \phi}{|\nabla \phi|}\right)=\operatorname{div}\left(\frac{\nabla \phi}{|\nabla \phi|}\right) \frac{\nabla \phi}{|\nabla \phi|}+\frac{1}{2} \nabla\left(\frac{\nabla \phi}{|\nabla \phi|} \cdot \frac{\nabla \phi}{|\nabla \phi|}\right)
$$

Since for a suitable level set representation we have $\frac{\nabla \phi}{|\nabla \phi|} \in L^{\infty}(D)$ and since the second term vanishes almost everywhere in $D$, condition (2.4) is satisfied if

$$
\operatorname{div}\left(\frac{\nabla \phi}{|\nabla \phi|}\right) \in L^{2}(D)
$$

Roughly speaking, the latter condition means that the curve has square-integrable curvature. Note that for a regular level set function with $|\nabla \phi| \geq c>0$ in $D$ we have due to the co-area formula

$$
c \int_{D}\left|\operatorname{div}\left(\frac{\nabla \phi}{|\nabla \phi|}\right)\right|^{2} d x \leq \int_{D}\left|\operatorname{div}\left(\frac{\nabla \phi}{|\nabla \phi|}\right)\right|^{2}|\nabla \phi| d x=\int_{\mathbb{R}} \int_{D \cap\{\phi=p\}} \kappa_{p}^{2} d s d p
$$

where $\kappa_{p}$ denotes the curvature of the level set $\{\phi=p\}$. 


\subsection{Variational Formulation and Weak Solutions}

The notion for a weak solution is obtained by writing (1.4) on each level set $\{\phi=p\}$, multiplying by a test function $v$ and integrating by parts. This implies, together with the coarea formula

$$
\begin{aligned}
0 & =\int_{\mathbb{R}} \int_{D \cap\{\phi=p\}}\left(-\operatorname{div}_{S}\left(\hat{a} \nabla_{S} u\right)+\hat{c} u-\hat{f}\right) v d s d p \\
& =\int_{\mathbb{R}} \int_{D \cap\{\phi=p\}}\left(\hat{a} \nabla_{S} u \cdot \nabla_{S} v+\hat{c} u v-\hat{f} v\right) d s d p \\
& =\int_{D}(\hat{a}(P \nabla u) \cdot(P \nabla v)+\hat{c} u v-\hat{f} v)|\nabla \phi| d x .
\end{aligned}
$$

We can define new coefficients via

$$
A:=\hat{a}|\nabla \phi|, \quad C=\hat{c}|\nabla \phi|, \quad F=\hat{f}|\nabla \phi|,
$$

which are equal to the original coefficients almost everywhere if $\phi$ is the signed distance function $b_{\Sigma}$ (since $\left|\nabla b_{\Sigma}\right|=1$ almost everywhere). Hence, weak solutions can be defined by

$$
\int_{D}(A(P \nabla u) \cdot(P \nabla v)+C u v-F v) d x=0, \quad \forall v \in H_{P}^{1} .
$$

If the coefficient $c$ is allowed to be zero, the solution of this variational problem is nonunique, since $u+\alpha$ is still a weak solution for any constant $\alpha$. Thus, we split the analysis into two cases, the first for $L^{2}$-coercive problems with strictly positive $c$ in Section 2.1, and the second for $c=0$ on a set of positive measure with the additional condition (1.9) in Section 2.2.

\section{$L^{2}$-coercive Problems}

In this section we consider problems with positive coefficients, i.e., we assume that there exist constants $\alpha_{0}>0$ and $\gamma_{0}>0$ such that

$$
a(S) \geq \alpha_{0}, \quad c(S) \geq \gamma_{0}, \quad \forall S \in \Sigma .
$$

Since the strict positivity of a function can be preserved in the extension to $D$, and since the function $\phi$ can always be chosen such that $\nabla \phi \in L^{\infty}(D)$ is bounded away from zero almost everywhere, we formulate the following assumptions on the extended coefficients:

Assumption $1 \mathrm{In}$ the remainder of this section we assume that $A, C$ and $F$ are elements of $L^{\infty}(D)$, and that there exist positive real constants $\alpha$ and $\gamma$ such that

$$
A(x) \geq \alpha, \quad C(x) \geq \gamma, \quad \forall x \in D .
$$

Under this assumption, we can define weak solutions in the following way:

Definition 1 An element $u \in H_{P}^{1}$ is a weak solution of (1.4), if it solves the variational problem

$$
B(u, v):=\int_{D}(A(P \nabla u) \cdot(P \nabla v)+C u v) d x=\int_{D} F v d x, \quad \forall v \in H_{P}^{1} .
$$

Under the above assumptions, the symmetric bilinear form $B$ in (2.8) is continuous and coercive on $H_{P}^{1} \times H_{P}^{1}$, i.e.,

$$
B(u, u)=\int_{D}(A(P \nabla u) \cdot(P \nabla v)+C u v) d x \geq \min \{\alpha, \gamma\}\|u\|_{H_{P}^{1}}^{2} .
$$

Thus, as a direct consequence of the Lax-Milgram Lemma, we may deduce the following result: 
Theorem 1 Let Assumption 1 be satisfied. Then there exists a unique weak solution (according to Definition 1) of the elliptic equation (1.4). Moreover, the weak solution of (1.4) is the unique minimizer of the functional

$$
J(v):=\int_{D}\left(\frac{A}{2}|P \nabla v|^{2}+\frac{C}{2} v^{2}-F v\right) d s
$$

The notion of weak solutions introduced by Definition 1 generalizes the concept of partial differential equations on surfaces to rather general shapes only satisfying (2.4). If the solution of the partial differential equation on the surface can be defined in a classical sense, then the two notions coincide, which we shall show in the following theorem:

Theorem 2 Let Assumption 1 be satisfied, let $\Sigma$ be rectifiable and let $\phi$ be chosen such that $\{\phi=p\} \cap D$ being rectifiable for almost every $p$. Then the trace of the weak solution $u$ according to Definition 1 and the solution $\tilde{u} \in H^{1}(\Sigma)$ of the elliptic equation (1.4) are equal.

Proof For each $p$ with $\Sigma^{p}:=\{\phi=p\} \cap D$ being rectifiable, there exists a unique solution $\tilde{u}^{p}$ of the variational problem

$$
J_{p}(v)=\int_{\Sigma^{p}}\left(\frac{a}{2}|\nabla v|^{2}+\frac{c}{2} v^{2}-f v\right) d s
$$

and $\tilde{u}^{0}$ equals the weak solution $\tilde{u} \in H^{1}(\Sigma)$ of (1.4). For each function $w \in C^{1}(D)$, we obtain that

$$
J_{p}\left(\tilde{u}^{p}\right) \leq J_{p}\left(\left.w\right|_{\Sigma^{p}}\right), \quad \text { for almost every } \mathrm{p} .
$$

Hence, the function $u \in H_{P}^{1}$ defined by $\left.u\right|_{\Sigma^{p}}=\tilde{u}^{p}$ satisfies

$$
J(u) \leq J(v), \quad \forall v \in C^{1}(D),
$$

and by a density argument even for all $v \in H_{P}^{1}(D)$. Theorem 1 implies that $u$ is the weak solution of the extension of (1.4) and thus, $\tilde{u}=\left.u\right|_{\Sigma}$.

Theorem 2 yields the consistency of the notion of weak solution induced by Definition 1 with the original solutions of (1.4), and as a consequence of this result, the solution on the surface does not depend on the level set function $\phi$. Another independence needed concerns the choice of the outer domain $D$ :

Theorem 3 Let $D_{1}$ and $D_{2}$ be two domains whose interior contains $\Sigma$, and let $u_{1}$ and $u_{2}$ denote the weak solutions of (1.4) on the domains $D_{1}$ and $D_{2}$, respectively. Then

$$
u_{1}=u_{2} \quad \text { on } D_{12}=\bigcup_{p,\{\phi=p\} \subset D_{1} \cap D_{2}}\{\phi=p\} .
$$

Proof Let $\hat{u}_{j}, j=1,2$ denote the extensions

$$
\hat{u}_{j}(x)= \begin{cases}u_{j}(x) & \text { if } x \in D_{12} \\ 0 & \text { if } x \in R^{d}-\overline{D_{12}}\end{cases}
$$

Since all jumps in $\hat{u}_{j}$ are orthogonal to the level sets of $\phi$, we obtain that

$$
P \nabla \hat{u_{j}}= \begin{cases}P \nabla u_{j}(x) & \text { if } x \in D_{12} \\ 0 & \text { if } x \in R^{d}-\overline{D_{12}} .\end{cases}
$$

Hence, $\hat{u}_{j}$ is an element of $H_{P}^{1}\left(D_{1}\right) \cap H_{P}^{1}\left(D_{2}\right)$ and we may use $v=\hat{u}_{1}-\hat{u}_{2}$ as an admissible test function in the bilinear form $B$ in (2.8) for both domains $D_{1}$ and $D_{2}$. As a direct consequence we obtain that

$$
0=\int_{D_{12}}\left(A\left|P \nabla\left(u_{1}-u_{2}\right)\right|^{2}+C\left(u_{1}-u_{2}\right)^{2}\right) d x \geq \gamma \int_{D_{12}}\left(u_{1}-u_{2}\right)^{2} d x
$$

and thus, $u_{1}=u_{2}$ a.e. in $D_{12}$. 
This result can be generalized to a stability estimate, which involves only localized information in terms of the levels of $\phi$ :

Theorem 4 Let Assumption 1 be satisfied and let $G: \mathbb{R} \rightarrow \mathbb{R}^{+} \cup\{0\}$ be a continuously differentiable function. Then the exists a unique weak solution $u$ of the elliptic equation (1.4) satisfies

$$
\int_{D} G(\phi)\left(|P \nabla v|^{2}+v^{2}\right) d s \leq C \int_{D} G(\phi) F^{2} d s
$$

for some positive real constant $C$.

Proof In the weak formulation we can use the test function $v=G(\phi) u$, which satisfies $P \nabla \phi=0$ a.e., and thus,

$$
P \nabla(G(\phi) u)=G^{\prime}(\phi) u P \nabla \phi+G(\phi) P \nabla u=G(\phi) P \nabla u .
$$

This implies

$$
B(u, v)=\int_{D} G(\phi)(A(P \nabla u) \cdot(P \nabla v)+C u v) d x \geq \min \{\alpha, \gamma\} \int_{D} G(\phi)\left(|P \nabla v|^{2}+v^{2}\right) d s,
$$

and

$$
B(u, v)=\int_{D} G(\phi) F u d x .
$$

An application of the Cauchy-Schwarz inequality thus implies the estimate for $C=\frac{1}{\min \{\alpha, \gamma\}}$.

Note that we can choose arbitrary smooth functions $G$ in Theorem 4, in particular those with local support around 0 , which implies in particular that the solution is independent of the extension of the parameters $A, C$, and $F$, away from $\Sigma$.

\section{Constrained Problems}

In the following we investigate problems without a zero order term, i.e., $c \equiv 0$, but with a mean constraint instead. For this sake we extend the mean constraint to all level sets via

$$
\int_{D} u v d x=0
$$

for all test functions $v$ such that $\left.v\right|_{\Sigma^{p}}$ is constant. This restriction of the test function $v$ is equivalent to $\nabla_{S} v=0$ on each $\Sigma^{p}$, respectively $P \nabla v=0$ in $D$. Thus, we may formulate a weak version of the mean constraint as

$$
\int_{D} u v d x=0, \quad \forall v \in H_{P, 0}^{1} .
$$

where

$$
H_{P, 0}^{1}:=\left\{v \in H_{P}^{1} \mid P \nabla v \equiv 0\right\} .
$$

We can now formulate the according variational problem as

$$
\frac{1}{2} \int_{D} A|P \nabla u|^{2} d x-\int_{D} F u d x \rightarrow \min _{u \in H_{P}^{1}}
$$

subject to (2.11).

By introducing the Lagrangian

$$
\mathcal{L}(u, p)=\frac{1}{2} \int_{D} A|P \nabla u|^{2} d x-\int_{D} F u d x+\int_{D} u p d x,
$$


we can derive an associated indefinite system for $u \in H_{P}^{1}, p \in H_{P, 0}^{1}$ given by

$$
\begin{array}{rlrl}
\int_{D} A(P \nabla u) \cdot \nabla v d x+\int_{D} v p d x & =\int_{D} F u d x & & \forall v \in H_{P}^{1} \\
\int_{D} u q d x & =0 & \forall q \in H_{P, 0}^{1} .
\end{array}
$$

For the analysis of the above problems, we introduce a standard assumption for this section: Assumption 2 In the remainder of this section we assume that $A$ and $F$ are elements of $L^{\infty}(D)$, and that there exists a positive real constants $\alpha$ such that

$$
A(x) \geq \alpha, \quad \forall x \in D .
$$

In order to show the well-posedness of the constrained problem, we need a Poincaré-type inequality, i.e., an estimate of the form

$$
\int_{D} u^{2} d x \leq C\left(\int_{D}|P \nabla u|^{2}+\left(\sup _{p \in H_{P, 0}^{1},\|p\|=1} \int_{D} p u d x\right)^{2}\right)
$$

for $u \in H_{P}^{1}$. For standard Sobolev spaces, such estimates are usually obtained using the compactness of the embedding into $L^{2}(D)$. Since the embedding from $H_{P}^{1}$ is the sum of a compact and a unitary operator, we cannot use similar arguments, but need a different type of proof. In the following we only give a result for smooth curves in $\mathbb{R}^{2}$, which we think could be generalized to less regular curves and surfaces with different methods from geometric measure theory:

Lemma 1 Let $\phi$ be such that the level sets $\Sigma^{p}=\{\phi=p\}$ are a union of $C^{1}$-curves for almost every $p$ and let $|\nabla \phi| \geq C$ for some positive constant $C$ almost everywhere. Then, for each $u \in$ $H_{P}^{1} \cap H_{P, 0}^{1}{ }^{\perp}$, the estimate

$$
\int_{D} u^{2} d x \leq \int_{D}|P \nabla u|^{2} d x
$$

holds.

Proof Since $C^{1}(D)$ is dense in $H_{P}^{1}$, it suffices to verify the estimate for $u \in C^{1}(D)$, the general result then follows from a standard closure argument. Due to the boundedness of $|\nabla \phi|$ and the co-area formula we have

$$
\int_{D} u^{2} d x \leq \frac{1}{C} \int_{D} u^{2}|\nabla \phi| d x=\frac{1}{C} \int_{\mathbb{R}} \int_{\Sigma^{p}} u^{2} d s d p
$$

Let $\Gamma \subset \Sigma^{p}$ be a $C^{1}$-curve, arc-length parametrized by $s \in[0, L]$. Then, for $s \in \Gamma, t \in \Gamma$ we have

$$
u(x(s))=u(x(t))+\int_{t}^{s} P \nabla u(\sigma) d \sigma
$$

and consequently

$$
u(x(s))=\frac{1}{L}\left(\int_{0}^{L} u(x(t)) d t+\int_{0}^{L} \int_{t}^{s} P \nabla u(\sigma) d \sigma d t\right)=\frac{1}{L} \int_{0}^{L} \int_{t}^{s} P \nabla u(\sigma) d \sigma d t .
$$

Thus,

$$
\int_{\Gamma} u^{2} d s \leq \frac{1}{L}\left(\int_{0}^{L}|P \nabla u(t)| d t\right)^{2} \leq \int_{0}^{L}|P \nabla u(t)|^{2} d t .
$$

The assertion now follows from inserting this inequality into the co-area formula. 
Theorem 5 Let Assumption 2 and the assumptions of Lemma 1 be satisfied. Then there exists a unique minimizer $\bar{u} \in H_{P}^{1}$ of the constrained optimization problem (2.13) subject to (2.11). Moreover, there exists a Lagrange multiplier $\bar{p} \in H_{P, 0}^{1}$, such that $(\bar{u}, \bar{p})$ is the unique solution of the indefinite problem (2.15), (2.16), and this solution depends continuously on $F$.

Proof In order to show the above result, we can use the standard theory of quadratic saddle-point problems (cf. [7]), which implies the assertion if there exist positive real constants $\alpha$ and $\beta$ such that

and

$$
\int_{D} A|P \nabla u|^{2} d x \geq \alpha\|u\|_{H_{P}^{1}}^{2}, \quad \forall u \in H_{P}^{1}, u \text { satisfies }(2.11)
$$

$$
\sup _{u \in H_{P}^{1}} \frac{\int_{D} u p d x}{\|u\|_{H_{P}^{1}}} \geq \beta\|p\|_{H_{P}^{1}}^{2}, \quad \forall p \in H_{P, 0}^{1} .
$$

The first follows from Lemma 1 and the latter from

$$
\sup _{u \in H_{P}^{1}} \frac{\int_{D} u p d x}{\|u\|_{H_{P}^{1}}} \geq \frac{\int_{D} p^{2} d x}{\|p\|_{H_{P}^{1}}}=\|p\|_{H_{P}^{1}} .
$$

\subsection{Finite Element Approximation}

In the following we consider the finite element approximation of the equation (1.4) by the extension to a domain $D$, which we assume to be polygonal (note that we are free to choose any form for the shape $D$ ). We assume that for grid-size $h>0, \mathcal{T}^{h}$ is a regular triangularization of $D$. By $\mathcal{V}^{h}$ we shall denote the according finite element space

$$
\mathcal{V}^{h}=\left\{v \in C(\bar{D})|v|_{T} \in \mathcal{P}^{k}(T), \forall T \in \mathcal{T}^{h}\right\}
$$

where $\mathcal{P}^{k}(T)$ is the set of polynomials of order less or equal to $k$ on $T$.

In the following we consider the standard finite element approximation of (2.8), which consists in finding $u^{h} \in \mathcal{V}^{h}$ such that

$$
B_{h}(u, v)=\int_{D} F_{h} v d x, \quad \forall v \in \mathcal{V}^{h},
$$

where $B_{h}$ and $F_{h} \in L^{2}(\Omega)$ are discrete approximations of the bilinear form $B$ and the right-hand side $F$ in (2.8). In a standard finite element approximation the discrete bilinear form arises due to discrete approximations of the coefficients and quadrature rules in the evaluation of integrals. We assume that $B_{h}$ is continuous on $\mathcal{V}^{h} \times \mathcal{V}^{h}$ and that there exist positive real constants $C_{h}$ such that

$$
B_{h}(u, u) \geq C_{h}\|u\|_{H_{P}^{1}} \times H_{P}^{1}, \quad \forall u \in \mathcal{V}^{h} .
$$

Under these assumptions on $B_{h}$, we may again apply the Lax-Milgram lemma to prove the well-posedness of the discretized problem (2.19):

Proposition 1 Under the above assumptions, there exists a unique solution $u^{h} \in \mathcal{V}^{h}$ of the variational problem (2.19).

As for any standard finite-element approximation of a coercive problem (cf. [6], we can obtain an approximation result of optimal order if $B_{h}=B$ :

Theorem 6 Let Assumption 1 be satisfied, and let $u$ be the unique solution of (1.4). Then there exists a constant $C>0$ such that

$$
\left\|u-u^{h}\right\|_{H_{P}^{1}} \leq C\left(\left\|F-F_{h}\right\|_{L^{2}(D)}+\inf _{v^{h} \in \mathcal{V}^{h}}\left\|u-v^{h}\right\|_{H_{P}^{1}}\right)
$$

where $u^{h} \in \mathcal{V}^{h}$ is the unique weak solution of (2.19). 
From the error estimate (2.20) one observes that the main factor influencing the approximation error is the projection error of the exact solution $u$ on the finite element subspace. In order to obtain an error estimate in terms of the discretization size $h$, one needs regularity of $u$ (and standard approximation results for finite element spaces). The regularity of $u$ is determined by two factors, namely the regularity of the problem on the original surface (i.e., of the coefficients and the surface itself) for derivatives in tangential directions and the regularity of the level set set function and coefficient extension for derivatives in normal direction. For the latter reason it becomes clear that the extension of the coefficients can influence the approximation order of the finite element method. The ideal extension of the solution would be a constant one in normal direction, since then the regularity of the extended solution corresponds exactly to the regularity of the solution on the surface, i.e., there is no loss of regularity.

In general, we have to expect that $B_{h}$ is of the form

$$
B_{h}(u, v)=\int_{D}\left(A_{h}\left(P_{h} \nabla u\right) \cdot\left(P_{h} \nabla v\right)+C_{h} u v\right) d x
$$

where $A_{h}, C_{h}$, and $P_{h}$ are discrete approximations of the coefficients and the projector $P$. Stability estimates depending on the errors $A-A_{h}$ and $C-C_{h}$ can be derived in standard norms, while there is in general no simple way to estimate errors when $P_{h} \neq P$. We shall therefore postpone studies with respect to discretizations of the level set function to future research.

As usual for finite element methods, one ends up with a finite-dimensional linear system of equations for the coefficients in the finite element representation after discretization. In the case of $L^{2}$-coercive problems, this linear system is of the form

$$
(\mathbf{M}+\mathbf{K}) \mathbf{u}=\mathbf{f},
$$

with matrices $\mathbf{M}, \mathbf{K} \in \mathbb{R}^{n \times n}$, and a right-hand side vector $\mathbf{f} \in \mathbb{R}^{n}$. The matrix $\mathbf{M}$ is a standard mass matrix, representing the discretization of the zero-order term, i.e., it is a positive definite matrix with eigenvalues positive and of order $h^{d}$, where $h$ denotes the discretization fineness. The matrix $\mathbf{K}$ is a stiffness matrix arising from the discretization of the differential operator, which is positive semidefinite, but which will have a nullspace in general, corresponding to the properties of the differential operator $-\operatorname{div}(A P \nabla u)$. On the other hand, the largest eigenvalue of $\mathbf{K}$ should be of order $h^{d-2}$. For moderate problem sizes, which can even be the case in 3D for appropriate grids with degrees of freedom concentrated along the surface, standard direct solution methods can be applied. If the problem size becomes very large, preconditioned iterative methods become inevitable. Since the system matrix is positive definite, a preconditioned conjugate gradient method is an obvious choice as an iterative solver. However, many of the standard preconditioning techniques for elliptic partial differential equations such as multigrid, multilevel, or domain decomposition methods are not successful in this case, because of the degeneracy and anisotropy of the differential operator. Therefore the only choices are either preconditioners based on imcomplete decompositions of the matrix $\mathbf{M}+\mathbf{K}$ (such as incomplete LU) or simple iterations such as Richardson or Gauss-Seidel. We shall numerically investigate the properties of such preconditioners below, an analysis and a construction of efficient preconditioners remains as a challenging task for future research (as in general for degenerate and anisotropic problems).

\section{Methods based on Phase-Field Representations}

In this section we discuss the approximation of (1.4) via finite element discretization of (1.7).

\subsection{Asymptotic Expansion}

In order to motivate the use of the phase-field representation, we start with a formal asymptotic expansion of (1.7), seeking a solution of the form

$$
v^{\epsilon}(x)=w_{0}(x)+\epsilon w_{1}(x)+\mathcal{O}\left(\epsilon^{2}\right) .
$$


We have $\psi=\mathcal{O}(1)$ and $\nabla \psi=\frac{1}{\epsilon} p^{\prime}\left(\frac{d}{\epsilon}\right)+\mathcal{O}(\epsilon)$. The corresponding expansion of (1.7) is given by

$$
\begin{aligned}
0 & =-\operatorname{div}\left(\left(1-\psi^{2}\right) \tilde{a} \nabla v^{\epsilon}\right)+\left(1-\psi^{2}\right) \tilde{c} v^{\epsilon}-\left(1-\psi^{2}\right) \tilde{f} \\
& =-2 \tilde{a} \frac{\psi}{\epsilon} p^{\prime}\left(\frac{d}{\epsilon}\right) \nabla d \cdot \nabla w_{0}+\left(1-\psi^{2}\right)\left[-\operatorname{div}\left(\tilde{a} \nabla w_{0}\right)+\tilde{c} w_{0}-\tilde{f}\right]+\mathcal{O}(\epsilon) .
\end{aligned}
$$

Hence, the leading order $\left(\epsilon^{-1}\right)$ term away from the interface $(\psi>>\epsilon)$ is proportional to $\nabla d \cdot \nabla w_{0}$ and since $\psi, p^{\prime}$, and $\tilde{a}$ are positive, we obtain that

$$
\nabla d \cdot \nabla w_{0}=0
$$

at leading order, which means that $w_{0}$ is constant in normal direction. From the second order expansion $\left(\epsilon^{0}\right)$ we obtain around the interface $\Sigma$ that

$$
-\operatorname{div}\left(\tilde{a} \nabla w_{0}\right)+\tilde{c} w_{0}=\tilde{f}
$$

and since $\nabla d \cdot \nabla w_{0}=0$, we can rewrite this equation as

$$
-\operatorname{div}\left(\tilde{a}(I-\nabla d \otimes \nabla d) \nabla w_{0}\right)+\tilde{c} w_{0}=\tilde{f} .
$$

Due to $|\nabla d|=1$, we have $(I-\nabla d \otimes \nabla d)=P$ and hence, the leading order term $w_{0}$ satisfies (1.4) at the interface $\Sigma$.

\subsection{Finite Element Approximation}

For the analysis of finite element schemes we use a basic assumption similar to the ones made in the level set case:

Assumption 3 In the remainder of this section we assume that $\tilde{a}, \tilde{c}$, and $\tilde{f}$ are elements of $L^{\infty}(D)$, and that there exist positive real constants $\alpha$ and $\gamma$ such that

$$
\tilde{a}(x) \geq \alpha, \quad \tilde{c}(x) \geq \gamma, \quad \forall x \in D .
$$

Moreover we assume that there exists $\alpha(\epsilon)>0$ such that

$$
-1+\beta(\epsilon) \leq \psi \leq 1-\beta(\epsilon),
$$

If Assumption 3 holds, then (1.7) is a nondegenerate elliptic equation, whose weak formulation (in the case of natural boundary conditions) is given by

$$
\int_{D}\left(1-\psi^{2}\right)(\tilde{a} \nabla v \nabla w+\tilde{c} v w-\tilde{f} w) d x=0
$$

for all test functions $w \in H^{1}(D)$. The finite element approximation of such a problem is a standard task, by choosing a subspace $\mathcal{V}^{h}$ as above, we just seek $v^{h} \in \mathcal{V}^{h}$ satisfying

$$
\int_{D}\left(1-\psi^{2}\right)\left(\tilde{a} \nabla v^{h} \nabla w+\tilde{c} v^{h} w-\tilde{f} w\right) d x=0 \quad \forall w \in \mathcal{V}^{h}
$$

The well-posedness of (3.5) and of the finite element discretization (3.6) can be carried out in a standard way via the Lax-Milgram lemma, using Assumption 3. Error estimates between the the solution $v^{\epsilon}$ of (3.5) and the solution $v^{\epsilon, h}$ of (3.6) for fixed $\epsilon$ can be derived, too. Together with an estimate between $v^{\epsilon}$ and a suitable extension of the solution $u$ on the surface one can then derive error estimates for the finite element approximation of the original partial differential equation. However, within this process one might loose order optimality of the estimates with respect to $\epsilon$, so that we consider a direct estimate between $v^{\epsilon, h}$ and $u$ in the following section.

Since the differential operator is neither degenerate nor anisotropic in this case, the solution of the arising discrete system can be carried out also by well-known iterative methods and preconditioners for elliptic partial differential equation. Some care is needed only due to the $\epsilon$-dependence of the condition number, which is caused by the fact that typically $\beta(\epsilon) \rightarrow 0$ as $\epsilon \rightarrow 0$. Noticing that we are interested in the solution at the surface only, it seems reasonable to use the energy norm for the stopping of the iterative methods, since in this way errors around the surface are weighted higher than errors far away. 


\subsection{Error Estimate}

In the following we derive an error estimate between the finite element solution $v^{\epsilon, h}$ of (3.6) and an extension of the solution $u$ of (1.4) on the surface. In order to keep the presentation reasonably simple we assume that $q \equiv 0$ in (1.3), that the surface $\Sigma$ is of class $C^{2}$ and that the extended coefficients $\tilde{a}, \tilde{c}$, and $\tilde{f}$ are $C^{1}$-functions. Since the above formal asymptotic expansion indicates that $v^{\epsilon}$ (and consequently $v^{\epsilon, h}$, too) is almost constant in normal direction, the natural extension of $u$ to choose for this sake is the one being constant in normal direction, which we denote by $\tilde{u}$.

As a norm suitable norm for the estimation of the error we use the rescaled norm

$$
\|\| v \|_{\epsilon}^{2}:=\frac{\int_{D}\left(1-\psi^{2}\right)\left(|\nabla v|^{2}+v^{2}\right) d x}{M_{\epsilon}}, \quad M_{\epsilon}=\int_{D}\left(1-\psi^{2}\right) d x .
$$

Note that, as $\epsilon \rightarrow 0$, we have

$$
\|v\|\left\|_{\epsilon} \rightarrow\right\| v \|_{H^{1}(\Sigma)}
$$

i.e., we approximate the desired norm on the surface. The projection of $\tilde{u}$ onto the finite element subspace $\mathcal{V}^{h}$ with the norm $\mid\|\cdot\| \|_{\epsilon}$ will be denoted by $\tilde{u}^{h}$.

In order to simplify notations, we introduce the bilinear form

$$
B_{\epsilon}(v, w):=\frac{1}{M_{\epsilon}} \int_{D}\left(1-\psi^{2}\right)(\tilde{a} \nabla v \cdot \nabla w+\tilde{c} v w) d x
$$

and the linear functional

$$
\left\langle F_{\epsilon}, w\right\rangle:=\frac{1}{M_{\epsilon}} \int_{D}\left(1-\psi^{2}\right) \tilde{f} w d x .
$$

Due to Assumption 3 the bilinear form $B_{\epsilon}$ is coercive and thus,

$$
\begin{aligned}
\min \{\alpha, \gamma\} \mid\left\|v^{\epsilon, h}-\tilde{u}\right\|_{\epsilon}^{2} & \leq B_{\epsilon}\left(v^{\epsilon, h}-\tilde{u}, v^{\epsilon, h}-\tilde{u}\right) \\
& =B_{\epsilon}\left(v^{\epsilon, h}, v^{\epsilon, h}-\tilde{u}^{h}\right)+B_{\epsilon}\left(v^{\epsilon, h}-\tilde{u}, \tilde{u}^{h}-\tilde{u}\right)-B_{\epsilon}\left(\tilde{u}, v^{\epsilon, h}-\tilde{u}^{h}\right) .
\end{aligned}
$$

Using the Cauchy-Schwarz inequality (in the scalar product corresponding to $\mid\|\cdot\| \|_{\epsilon}$ ) for the second term we obtain the existence of some constant $C_{0}>0$ (independent of $\epsilon$ ) such that

$$
\|\| v^{\epsilon, h}-\tilde{u}\left\|\left.\right|_{\epsilon} ^{2} \leq B_{\epsilon}\left(v^{\epsilon, h}, v^{\epsilon, h}-\tilde{u}^{h}\right)-B_{\epsilon}\left(\tilde{u}, v^{\epsilon, h}-\tilde{u}^{h}\right)+C_{0}\left|\left\|\tilde{u}^{h}-\tilde{u}\right\|\right|_{\epsilon}^{2} .\right.
$$

Moreover, since $v^{\epsilon, h}-\tilde{u}^{h} \in \mathcal{V}^{h}$, it is an admissible test function in (3.6), which implies

$$
B_{\epsilon}\left(v^{\epsilon, h}, v^{\epsilon, h}-\tilde{u}^{h}\right)=\left\langle F_{\epsilon}, v^{\epsilon, h}-\tilde{u}^{h}\right\rangle .
$$

Hence, the remaining term to estimate is

$$
\left\langle F_{\epsilon}, v^{\epsilon, h}-\tilde{u}^{h}\right\rangle-B_{\epsilon}\left(\tilde{u}, v^{\epsilon, h}-\tilde{u}^{h}\right)=-\frac{1}{M_{\epsilon}} \int_{D}\left(1-\psi^{2}\right)\left(\tilde{a} \nabla \tilde{u} \cdot \nabla \tilde{w}^{h}+\tilde{c} \tilde{u} w^{h}-\tilde{f} w^{h}\right) d x
$$

with $w^{h}=v^{\epsilon, h}-\tilde{u}^{h}$.

Now we split the domain $D$ into two parts, namely into

$$
D_{\epsilon}=\{x \in D|| d(x) \mid \leq \delta(\epsilon)\}
$$

and its complement. Since $\tilde{u}$ is constant in normal direction, i.e., $\nabla d \cdot \nabla \tilde{u}=0$, we obtain with $P=(I-\nabla d \otimes \nabla d)$ that

$$
\begin{aligned}
& -\int_{D_{\epsilon}}\left(1-\psi^{2}\right)\left(\tilde{a} \nabla \tilde{u} \cdot \nabla \tilde{w}^{h}+\tilde{c} \tilde{u} w^{h}-\tilde{f} w^{h}\right) d x \\
& =-\int_{D_{\epsilon}}\left(1-\psi^{2}\right)\left(\tilde{a} P \nabla \tilde{u} \cdot \nabla \tilde{w}^{h}+\tilde{c} \tilde{u} w^{h}-\tilde{f} w^{h}\right) d x \\
& =-\int_{-\delta(\epsilon)}^{\delta(\epsilon)}\left(1-p(t / \epsilon)^{2}\right) \int_{\{d=t\}}\left(\tilde{a} \nabla_{S} \tilde{u} \nabla_{S} w^{h}+\tilde{c} \tilde{u} w^{h}-\tilde{f} w^{h}\right) d S d t .
\end{aligned}
$$


Using that $\tilde{u}$ is the extension of a solution on the surface $\Sigma$, i.e.,

$$
\int_{\{d=0\}}\left(\tilde{a} \nabla_{S} \tilde{u} \nabla_{S} w^{h}+\tilde{c} \tilde{u} w^{h}-\tilde{f} w^{h}\right) d S=0,
$$

together with the smoothness of the extensions, one can derive (after tedious work) an estimate of the form

$$
-\int_{\{d=t\}}\left(\tilde{a} \nabla_{S} \tilde{u} \nabla_{S} w^{h}+\tilde{c} \tilde{u} w^{h}-\tilde{f} w^{h}\right) d S \leq C t\left\|w^{h}\right\|_{H^{1}(\{d=t\})}
$$

with a constant $C>0$, provided $\delta(\epsilon)$ is sufficiently small. Hence, by applying further the CauchySchwarz inequality and the co-area formula we deduce

$$
\begin{aligned}
& -\int_{D_{\epsilon}}\left(1-\psi^{2}\right)\left(\tilde{a} \nabla \tilde{u} \cdot \nabla \tilde{w}^{h}+\tilde{c} \tilde{u} w^{h}-\tilde{f} w^{h}\right) d x \\
& \quad \leq C \int_{-\delta(\epsilon)}^{\delta(\epsilon)}\left(1-p(t / \epsilon)^{2}\right) t\left\|w^{h}\right\|_{H^{1}(\{d=t\})} d t \\
& \quad \leq C \delta(\epsilon)\left(\int_{-\delta(\epsilon)}^{\delta(\epsilon)}\left(1-p(t / \epsilon)^{2}\right)\left\|w^{h}\right\|_{H^{1}(\{d=t\})}^{2} d t\right)^{1 / 2} \\
& \quad \leq C \delta(\epsilon)\left(\int_{D_{\epsilon}}\left(1-\psi^{2}\right)\left(\left|P \nabla w^{h}\right|^{2}+\left|w^{h}\right|^{2}\right) d x\right)^{1 / 2} \\
& \quad \leq C \delta(\epsilon) \sqrt{M_{\epsilon}}\left|\left\|w^{h}\right\|\right|_{\epsilon} .
\end{aligned}
$$

On the remaining part $D \backslash D_{\epsilon}$ we simply use the Cauchy-Schwarz inequality, boundedness of the extensions, and monotonicity of $p$ to estimate

$$
-\int_{D_{\epsilon}}\left(1-\psi^{2}\right)\left(\tilde{a} \nabla \tilde{u} \cdot \nabla \tilde{w}^{h}+\tilde{c} \tilde{u} w^{h}-\tilde{f} w^{h}\right) d x \leq C \sqrt{1-p(\delta(\epsilon) / \epsilon)^{2}} \sqrt{M_{\epsilon}} \mid\left\|w^{h}\right\| \|_{\epsilon}
$$

Finally, we can use that

$$
\left|\left\|w^{h}\right\|\right|_{\epsilon} \leq\left|\left\|v^{\epsilon, h}-\tilde{u}\right\|\right|_{\epsilon}+\left|\left\|u^{h}-\tilde{u}\right\|\right|_{\epsilon}
$$

in all the estimates in order to reduce to terms that can be estimated.

Combining all the estimates above, we obtain the following result:

Theorem 7 Let Assumption 3 and the above smoothness assumptions be satisfied. Then, for $\delta(\epsilon)$ and $\epsilon$ sufficiently small, the estimate

$$
\left|\left\|v^{\epsilon, h}-\tilde{u}\right\|\right|_{\epsilon} \leq C\left(\left|\left\|u^{h}-\tilde{u}\right\|\right|_{\epsilon}+\frac{\sqrt{1-p(\delta(\epsilon) / \epsilon)^{2}}+\delta(\epsilon)}{\sqrt{M_{\epsilon}}}\right)
$$

holds for some constant $C>0$ independent of $\epsilon$.

The first term in (3.7) depends mainly on the order of the finite element approximation, if $\epsilon$ is chosen appropriately in dependence of $h$, then one can still expect

$$
\left|\left\|u^{h}-\tilde{u}\right\|\right|_{\epsilon}=\mathcal{O}\left(h^{k}\right)
$$

for standard polynomial elements of order $k$. The second term is a measure for the approximation quality of the phase-field representation rather than the finite element method. In general we can expect $M_{\epsilon}=\mathcal{O}(\epsilon)$ and hence, we need

$$
\frac{\delta(\epsilon)}{\sqrt{\epsilon}} \rightarrow 0, \quad \frac{1-p(\delta(\epsilon) / \epsilon)^{2}}{\epsilon} \rightarrow 0 .
$$

The latter can hold only if $\frac{\epsilon}{\delta(\epsilon)} \rightarrow 0$, i.e., a suitable choice is $\delta(\epsilon) \sim \epsilon^{\rho}$, with $\rho \in\left(\frac{1}{2}, 1\right)$. The optimal choice of course depends on the specific form of the phase-field function $p$, but in any case the approximation order is lower than $\mathcal{O}(\sqrt{\epsilon})$, which is a bound for the order of $\frac{\delta(\epsilon)}{\sqrt{M_{\epsilon}}}$. 


\section{Numerical Tests}

In the following we report the results for some numerical tests with different geometries. Due to the extension of the interface equation to a domain $D \subset \mathbb{R}^{d}$, one can use standard finite element implementations. We realized all the computational examples below within the finite element package FEMLAB 3.

\subsection{Single Circle}

Our first numerical example is the solution of the equation

$$
-\frac{\partial^{2} u}{\partial S^{2}}+u=2 \cos (S), \quad S \in(-\pi, \pi)
$$

on the unit circle. Here we can identify the surface parametrization $S$ with the angle between a point on the surface and the unit vector $(1,0)^{T}$. The unique solution of this problem can be computed explicitely as

$$
\tilde{u}=\cos (S),
$$

and we can use this exact solution for the quantitative validation of solvers.

We solve the extended problem on a domain of the form $D=(-L, L)^{2}$, with $L=1.5$ unless further noticed. The level set function $\phi$ is simply given by the signed distance function in this case, which can be computed explicitely as

$$
d(x)=\sqrt{x_{1}^{2}+x_{2}^{2}}-1 .
$$

We first test the numerical convergence and convergence rate. For this sake we use piecewise linear and piecewise quadratic finite elements and compute the error between the finite element solution and the exact solution in the norms of $L^{2}(\Sigma)$ and $H^{1}(\Sigma)$. More precisely, we solve the discretized problem on a regular grid of width $h$ (starting from $h=\frac{1}{16}$ and subsequently dividing $h$ by two) and evaluate the finite element solution and its gradient on a Lagrangian grid on the unit sphere (of width $10^{-6} \pi$ ), where we numerically compute the error by quadrature. The results are presented in Table 1 for piecewise linear and in Table 2 for piecewise quadratic finite elements. In both cases, one observes that the error in both norms decreases with the grid size. Since the grid size $h_{k}$ is divided by two to obtain $h_{k+1}$, we can compute the numerical convergence rate as

$$
r_{k}=\frac{1}{\log (2)}\left(\log e_{k}-\log e_{k+1}\right),
$$

where $e_{k}$ denotes the error obtained with grid size $h_{k}$. One observes that the convergence rate in the $H^{1}$-norm equals the order of the finite elements, i.e. $r_{k} \approx 1$ for piecewise linear and $r_{k} \approx 2$ for piecewise quadratic elements. This confirms our error estimates of optimal order, numerically the order seems not only to hold for the extension to $D$, but also for the restriction of $u$ to $\Sigma$.

We also demonstrate that the degrees of freedom can be concentrated along the curve. For this sake we use an adapted grid around the circle, as shown in Figure 1, with a total number of 6142 degrees of freedom for piecewise linear elements. The grid fineness around the curve is around $h=\frac{1}{500}$. A color plot of the corresponding solution is given in the left part of Figure 1 . The errors between exact and finite element solution are given by

$$
\left\|u^{h}-\tilde{u}\right\|_{L^{2}(\Sigma)}=4.12 e-4, \quad\left\|u^{h}-\tilde{u}\right\|_{H^{1}(\Sigma)}=4.69 e-4
$$

i.e., the $L^{2}$-error is compareable to the one obtained with a regular grid at $h=\frac{1}{256}$ (more than $10^{5}$ degrees of freedom) and the $H^{1}$-error is even less than on the regular grid at $h=\frac{1}{1024}$ (more than $2 \times 10^{6}$ degrees of freedom). 


\begin{tabular}{|r|r|r|r|r|}
\hline$h$ & $\left\|u^{h}-\tilde{u}\right\|_{L^{2}(\Sigma)}$ & $L^{2}$-Rate & $\left\|u^{h}-\tilde{u}\right\|_{H^{1}(\Sigma)}$ & $H^{1}$-Rate \\
\hline $1 / 16$ & $0.27 \mathrm{e}-1$ & & $0.46 \mathrm{e}-1$ & \\
$1 / 32$ & $0.15 \mathrm{e}-1$ & 0.85 & $0.28 \mathrm{e}-1$ & 0.71 \\
$1 / 64$ & $0.44 \mathrm{e}-2$ & 1.77 & $0.19 \mathrm{e}-1$ & 0.56 \\
$1 / 128$ & $0.59 \mathrm{e}-3$ & 2.90 & $0.85 \mathrm{e}-2$ & 1.16 \\
$1 / 256$ & $0.24 \mathrm{e}-4$ & 4.62 & $0.35 \mathrm{e}-2$ & 1.29 \\
$1 / 512$ & $0.14 \mathrm{e}-4$ & 0.78 & $0.18 \mathrm{e}-2$ & 0.96 \\
$1 / 1024$ & $0.32 \mathrm{e}-5$ & 2.13 & $0.91 \mathrm{e}-3$ & 0.98 \\
\hline
\end{tabular}

Table 1 Numerical error and convergence rates in the norms $L^{2}(\Sigma)$ and $H^{1}(\Sigma)$ in example one, obtained with piecewise linear finite elements and the level set representation.

\begin{tabular}{|r|r|r|r|r|}
\hline$h$ & $\left\|u^{h}-\tilde{u}\right\|_{L^{2}(\Sigma)}$ & $L^{2}$-Rate & $\left\|u^{h}-\tilde{u}\right\|_{H^{1}(\Sigma)}$ & $H^{1}$-Rate \\
\hline $1 / 16$ & $0.34 \mathrm{e}-2$ & & $0.13 \mathrm{e}-1$ & \\
$1 / 32$ & $0.56 \mathrm{e}-3$ & 2.60 & $0.24 \mathrm{e}-2$ & 2.44 \\
$1 / 64$ & $0.85 \mathrm{e}-4$ & 2.72 & $0.33 \mathrm{e}-3$ & 2.86 \\
$1 / 128$ & $0.24 \mathrm{e}-5$ & 5.15 & $0.70 \mathrm{e}-4$ & 2.23 \\
$1 / 256$ & $0.43 \mathrm{e}-7$ & 5.80 & $0.17 \mathrm{e}-4$ & 2.04 \\
$1 / 512$ & $0.54 \mathrm{e}-8$ & 2.99 & $0.44 \mathrm{e}-5$ & 1.95 \\
\hline
\end{tabular}

Table 2 Numerical error and convergence rates in the norms $L^{2}(\Sigma)$ and $H^{1}(\Sigma)$ in example one, obtained with piecewise quadratic finite elements and the level set representation.

Fig. 1 Solution on circle using an adaptive mesh (left) and corresponding mesh (right).

\begin{tabular}{|r|r|r|r|r|r|}
\hline$h$ & $\epsilon$ & $\left\|u^{h}-\tilde{u}\right\|_{L^{2}(\Sigma)}$ & $L^{2}$-Rate & $\left\|u^{h}-\tilde{u}\right\|_{H^{1}(\Sigma)}$ & $H^{1}$-Rate \\
\hline $1 / 16$ & 0.05 & $0.85 \mathrm{e}-2$ & & $0.48 \mathrm{e}-1$ & \\
$1 / 32$ & 0.02 & $0.23 \mathrm{e}-2$ & 1.88 & $0.26 \mathrm{e}-1$ & 0.88 \\
$1 / 64$ & 0.02 & $0.21 \mathrm{e}-2$ & 0.13 & $0.13 \mathrm{e}-1$ & 1.00 \\
$1 / 128$ & 0.01 & $0.19-2$ & 0.14 & $0.64 \mathrm{e}-2$ & 1.02 \\
$1 / 256$ & 0.01 & $0.17 \mathrm{e}-2$ & 0.16 & $0.35 \mathrm{e}-2$ & 0.87 \\
$1 / 512$ & 0.01 & $0.17 \mathrm{e}-2$ & 0.00 & $0.24 \mathrm{e}-2$ & 0.54 \\
\hline
\end{tabular}

Table 3 Numerical error and convergence rates in the norms $L^{2}(\Sigma)$ and $H^{1}(\Sigma)$ in example one, obtained with piecewise linear finite elements and the phase-field representation.

For exactly the same problem we also present the results of the phase-field approach with phase field function

$$
\psi=1-\frac{2}{1+\exp (-d / \epsilon)},
$$

using linear finite elements on a regular grid. The resulting errors for different discretization levels (with appropriate choice of $\epsilon=\epsilon(h)$ ) are given in Table 3. One observes that the magnitude of the errors is very close to the ones in the level set case. Moreover, the expected order of convergence in terms of $h$ is achieved well numerically in the $H^{1}$-norm.

In order to compare the effect of the different extensions, we plot the solutions obtained with the level set and the phase-field representation in Figure 2. From this plot one clearly observes a difference, the phase-field solution (right) is almost constant in normal direction as predicted by the formal asymptotic expansion, whereas the level set solution (left) varies strongly in normal direction. However, note that the normal variation of the level set solution relies on the specific extension of the coefficients, an extension like in [21] would yield a solution that is constant in normal direction. 


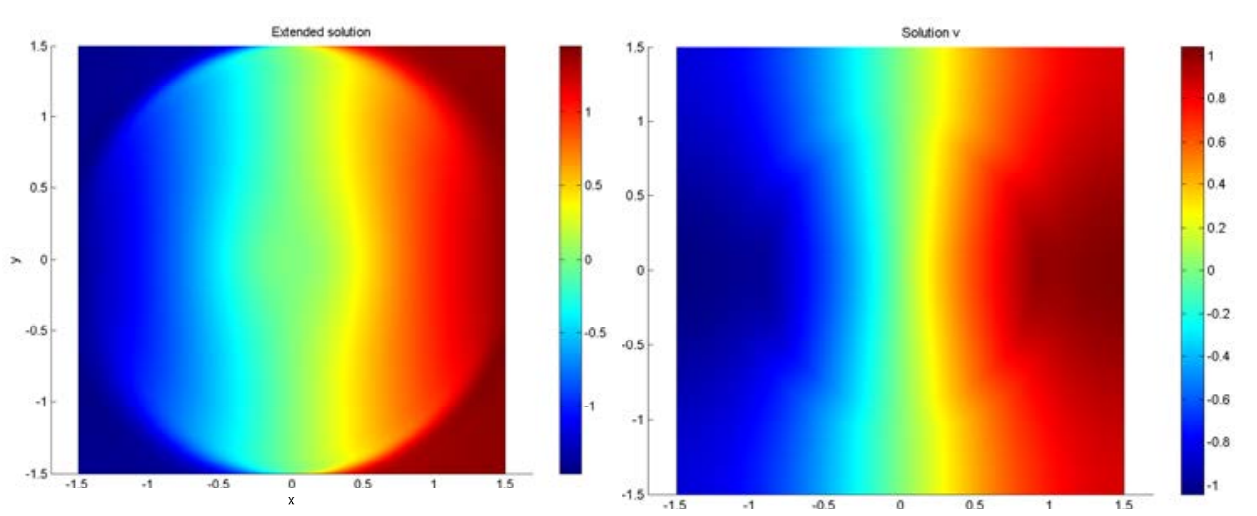

Fig. 2 Numerical solution obtained for level set (left) and phase-field representation (right), $h=\frac{1}{64}$.

\subsection{Sphere}

Our second example is the solution of (1.4) on a sphere of radius 0.7 . We use the setting $c=1$ and the right-hand side

$$
f(x, y, z)=\frac{x y}{x^{2}+y^{2}+z^{2}}
$$

The coefficient $a$ is chosen constant and varied between 1 and $10^{-4}$, the latter representing an approximation to a solution of the Laplace-Beltrami equation with the constraint of mean zero for the solution.

The solutions obtained with piecewise linear finite elements on a grid with 185995 nodes are illustrated in Figure 3 for the two different representations in the case $a=10^{-4}$. Both methods behave very similar in this case, their difference is very small and hardly visible even on coarse grids.

As noticed above, the iterative solution of the discretized problems is not a simple task, in particular in the case of the level set representation. For this sake we compare iteration numbers obtained with the preconditioned conjugate gradient method. The preconditioners used are SSOR relaxation, incomplete LU-decomposition (ILU), and geometric multigrid (GMG). The results are given in Table 4, with the notation $\mathrm{LS} / *$ for level set representations and $\mathrm{PF} / *$ for phase-field representations using the respective preconditioner, the specified accuracy in the residual being $10^{-6}$. Due to the degeneracy of the level set problem it is no surprise that multigrid preconditioners did not yield reasonable iteration numbers and even failed to converge within 10000 iterations except on coarse grids. For small $a$, the iteration numbers with SSOR and ILU preconditioning are very similar, whereas the latter behaves significantly better as $a$ increases. In any of the cases one observes that the iteration numbers depend on $h$, but at least they do not grow too fast with respect to the number of unknowns and the efficiency is not much worse than for standard elliptic problems with these kind of preconditioners. For the phase-field representation the situation differs completely, here ILU preconditioning produces by far the highest numbers of iterations, SSOR preconditioning yields similar (but lower) iteration numbers than in the level set case. The geometric multigrid preconditioner yields a low number of almost $h$-independent iteration numbers as expected. It is remarkable however, that this $h$-independence remains true in our test examples not only for fixed $\epsilon$, but also when $\epsilon$ is decreased appropriately with $h$ (also in the results reported in Table 4). 


\begin{tabular}{|r|r|r|r|r|r|}
\hline \# of elements & $a$ & It. LS/SSOR & It. LS/ILU & It. PF/SSOR & It. PF/GMG \\
\hline 16879 & $1 \mathrm{e}-4$ & 10 & 28 & 24 & 5 \\
59659 & $1 \mathrm{e}-4$ & 49 & 48 & 39 & 6 \\
185995 & $1 \mathrm{e}-4$ & 102 & 116 & 60 & 6 \\
583325 & $1 \mathrm{e}-4$ & 175 & 197 & 88 & 6 \\
16879 & $1 \mathrm{e} 0$ & 29 & 20 & 23 & 5 \\
59659 & $1 \mathrm{e} 0$ & 36 & 30 & 35 & 6 \\
185995 & $1 \mathrm{e} 0$ & 80 & 45 & 52 & 6 \\
583325 & $1 \mathrm{e} 0$ & 126 & 68 & 76 & 6 \\
\hline
\end{tabular}

Table 4 Iteration numbers for elliptic equation on sphere with different preconditioning strategies.
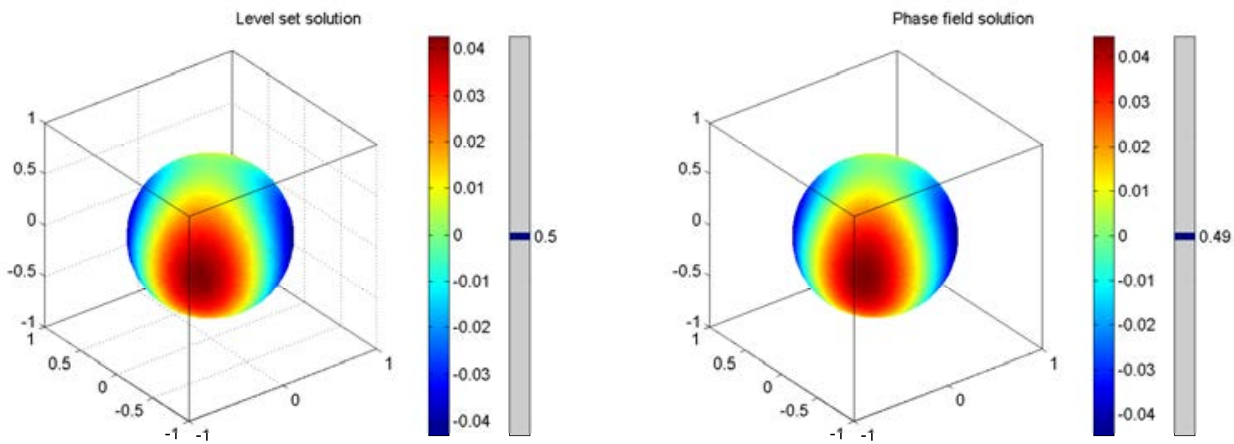

Fig. 3 Numerical solution on the sphere obtained for level set (left) and phase-field representation (right).

\subsection{Torus}

Our final example is the solution of (1.4) with $c=1, a=10^{-4}$, and the same $f$ as in the last example, but this time on a torus specified by the level set function

$$
\phi(x, y, z)=\sqrt{\left(\sqrt{x^{2}+y^{2}}-0.6\right)^{2}+z^{2}}-0.25 .
$$

The observed behaviour with respect to discretization fineness and iteration numbers for the solution of the arising discrete system as in the examples before, the main difference being a higher grid resolution needed due to higher curvates on the torus surface.

The results for obtained with the level set representation and the phase field representation $(\epsilon=0.1)$ on a grid with around 500.000 tetraeders are illustrated in Figure 4. The main difference that can be observed is that the maxima of the level set solution are slightly higher than the maxima of the phase-field solution, which might be due to additional dissipation in the scheme based on phase-field representation.

\section{Acknowledgments}

A major part of this work was carried out when the author was at the Department of Mathematics, UCLA. I would like to thank Stanley Osher (UCLA) for stimulating this research and fruitful discussions on the subject, and Wolfgang Ring (University Graz), Joachim Schöberl and Sven Beuchler (RICAM and University Linz) for useful and stimulating discussions. Financial support is acknowledged by the Austrian National Science Foundation FWF through project SFB F 013 / 08, the Johann Radon Institute for Computational and Applied Mathematics (Austrian Academy of Sciences ÖAW), the NSF through ITR grant ACI-0321917, and the ONR through grant N0001402-1-0720. 

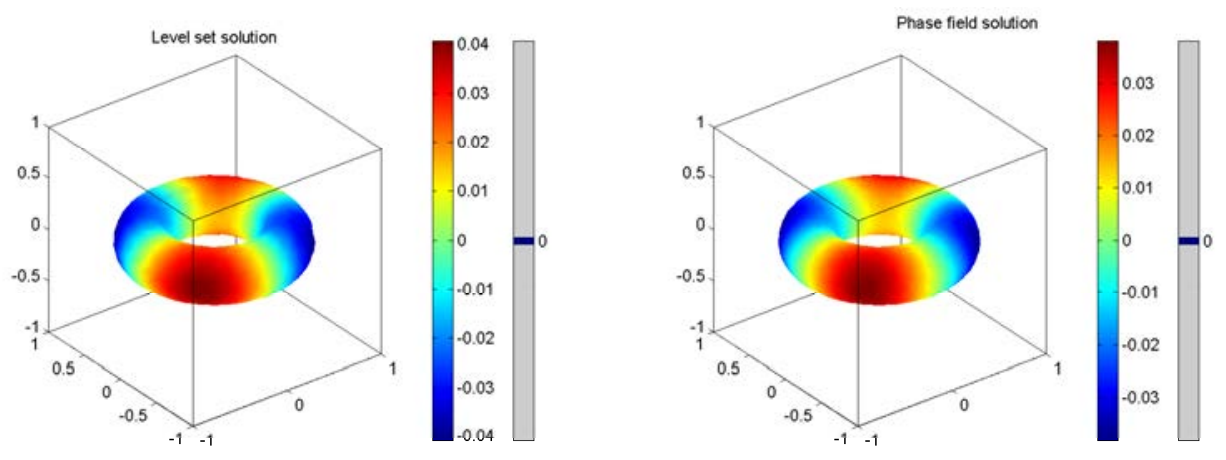

Fig. 4 Numerical solution on the torus obtained for level set (left) and phase-field representation (right).

\section{References}

1. R.A.Adams, Sobolev Spaces (Academic Press, New York, London, 1975).

2. G.Bal, K.Ren, Reconstruction of singular surfaces by shape sensitivity analysis and level set methods, Preprint (Columbia University, 2005).

3. G.Barles, H.M.Soner, P.E.Souganidis, Front propagation and phase field theory, SIAM J. Control Optim. 31 (1993), 439-469.

4. M.Bertalmio, L.T.Cheng, S.Osher, G.Sapiro, Variational problems and partial differential equations on implicit surfaces: The framework and examples in image processing and pattern formation, J. Comput. Phys. 174 (2001), 759-780.

5. M.Bertalmio, F.Memoli, L.T.Cheng, G.Sapiro,S.Osher, Variational problems and partial differential equations on implicit surfaces: Bye bye triangulated surfaces? in: S.Osher, N.Paragios, eds., Geometric Level Set Methods in Imaging, Vision, and Graphics (Springer, New York, 2003), 381-398.

6. S.Brenner, L.R.Scott, The Mathematical Theory of Finite Element Methods (2nd edition, Springer, Berlin, 2002).

7. F.Brezzi, M.Fortin, Mixed and Hybrid Finite Element Methods, Springer Series in Computational Mathematics 15 (Springer, New York, 1991).

8. M.Burger, A framework for the construction of level set methods for shape optimization and reconstruction, Interfaces and Free Boundaries 5 (2003), 301-329.

9. M.Burger, Surface diffusion including adatoms, CAM-Report 04-64 (UCLA, 2004), and submitted.

10. J.W.Cahn, J.E.Taylor, Surface motion by surface diffusion, Acta Metall. Mater. 42 (1994), 1045-1063.

11. G.Caginalp, E.Socolovsky, Phase field computations of single-needle crystals, crystal growth, and motion by mean curvature, SIAM J. Sci. Comput. 15 (1994), 106-126.

12. F.Davi, M.E.Gurtin, On the motion of a phase interface by surface diffusion, J. Appl. Math. Phys. 41 (1990), 782-811.

13. K.Deckelnick, C.M.Elliott, V.Styles, Numerical diffusion induced grain boundary motion, Interfaces and Free Boundaries 3 (2001), 393-414

14. M.C.Delfour, J.P.Zolésio,Shapes and geometries. Analysis, differential calculus, and optimization (SIAM, Philadelphia, 2001).

15. U.Diewald, T.Preusser, M.Rumpf, Anisotropic diffusion in vector field visualization on Euclidean surfaces, IEEE Trans. Vis. Comp. Graph. 6(2000), 139-149.

16. J.Dorsey, P.Hanrahan, Digital materials and virtual weatherings, Scient. Amer. 282:2 (2000), 46-53.

17. L.C. Evans, Partial Differential Equations, AMS Graduate Studies in Mathematics 19 (AMS, Providence, 1998).

18. P.C.Fife, J.W.Cahn, C.M.Elliott, A free boundary model for diffusion induced grain boundary motion, Interfaces and Free Boundaries 3 (2001), 291-336.

19. M.Hintermüller, W.Ring, An Inexact Newton-CG-type active contour approach for the minimization of the Mumford-Shah functional, J. Math. Imag. Vision 20 (2004), 19-42.

20. O.Faugeras, F.Clément, R.Deriche, R.Keriven, T.Papadopoulo, J.Roberts, T.Viville, F.Devernay, J.Gomes, G.Hermosillo, P.Kornprobst, D.Lingrand, The inverse EEG and MEG problems, the adjoint state approach, I: The continuous case Research Report RR-3673 (INRIA, 1999).

21. J.B.Greer, An improvement of a recent Eulerian method for solving PDEs on general geometries, CAM-Report 05-41 (UCLA, 2005).

22. J.B.Greer, A.L.Bertozzi, G.Sapiro, Fourth order partial differential equations on general geometries, CAM-Report 05-41 (UCLA, 2005). 
23. J.L.Lions, E.Magenes, Non-Homogeneous Boundary Value Problems and Applications (Springer, Berlin, Heidelberg, New York, 1972)

24. S.J.Osher, R.P.Fedkiw, The Level Set Method and Dynamic Implicit Surfaces (Springer, New York, 2002).

25. S.J.Osher, J.A.Sethian, Fronts propagating with curvature-dependent speed: algorithms based on Hamilton-Jacobi formulations, J. Comp. Phys. 79 (1988), 12-49.

26. G.Turk, Generating textures on arbitrary surfaces using reaction-diffusion, Computer Graphics 25 (1991), 289-298. 